Modeling Cross-Field Drifts and Current With the B2 Code for the CIT Divertor

T.D. Rognlien

J.L. Mijovich

I1. E. Rensink

November 8, 1990

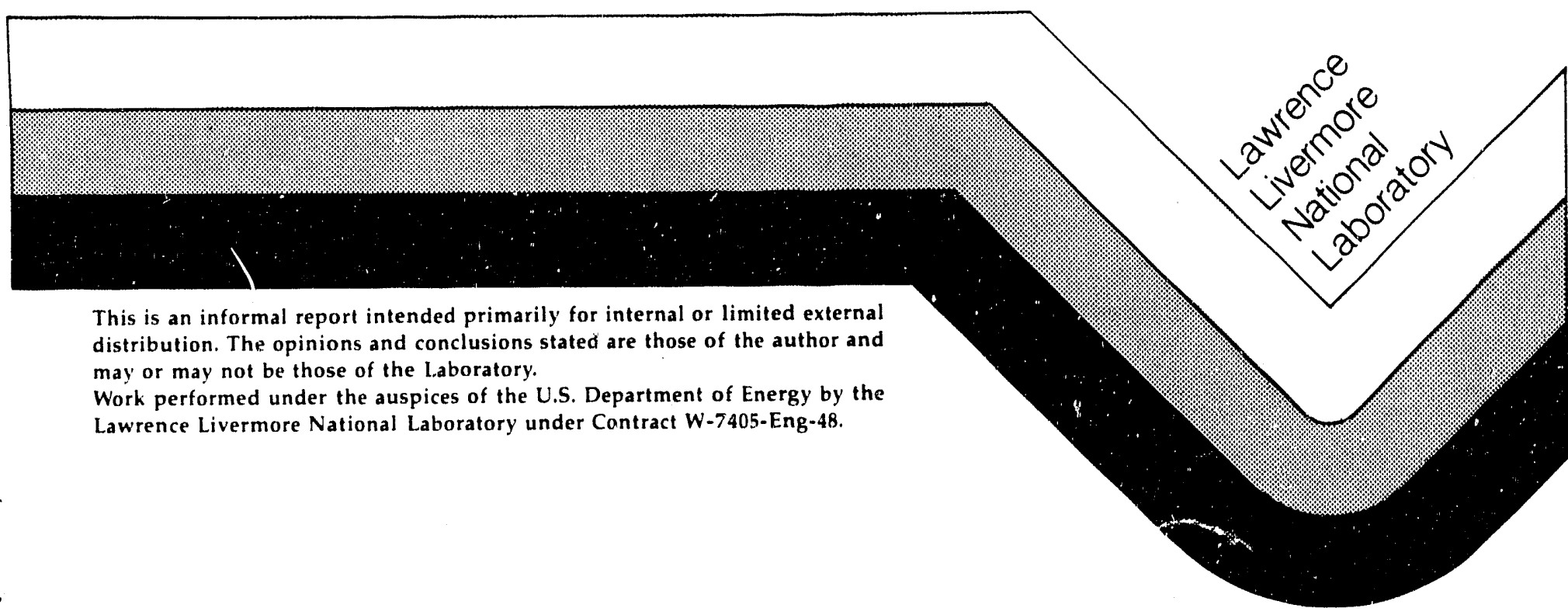


This document was prepared as an account of work sponsored by an agency of the United Slates Covernment. Neither the United States Government not the University of California nor any of their employees, makes any warranty, express or implied. or assumes any legal liability or responsibility for the accuracy, completeness, or usefulness of any information, apparatus, product, or process disclosed, or represents that its use would not infringe privately owned rights. Reference herein to any specific commercial products, process, or service by trade name, trademark, manufacturet, or otherwise, does not necessarily constitute or imply its endorsement, recommendation, or favoring by the United States Government or the University of California. The views and opinions of authors expr'sied herein do not necessarily state or reflect those of the United States Government or the University of California, and shall not be used for advertising or product endorsement purposes.

This report has been reproduced

directly from the best available copy.

Available to DOE and DOr. ontractors front the

Office of Scientific and Technical Information

P.O. Box 62 Oak Ridze. TN 37831

Prices avalable from (615) 576-8401, FTS (- $6-6401$.

Avallable to the public from the

National Technical Information Service

U.S. Department of Commerce

5285 Port Royal Rd.,

Springfield, VA 2216

Price

Code

A01

Papercopy Prices

$\mathrm{A02}$

$\mathrm{A03}$

A04

A05

A06

A07

A08

A09

A10

A11

A12

A13

A14

A15

A16

A17

A18

A19

A20

A21

A22

A23

A24

A25

A99
Page

Range

Microfiche 


\title{
MODELING CROSS-FIELD DRIFTS AND CURRENT WITH THE B2 CODE FOR THE CIT DIVERTOR
}

\author{
A Status Report for the CIT Project \\ October 12,1990 \\ UCRL - ID- -105272 \\ T. D. Rognlien, J. L. Milovich, and M. E. Rensink \\ Lawrence Livermore National Laboratory \\ University of California, Livermore, CA 94550 \\ DE91 004948
}

\section{INTRODUCTION}

We have modified the B2 edge-plasma code ${ }^{1}$ to include the effects of classical fluid drifts across the magnetic field lines and plasma currents. This report presents preliminary results of these effects for the CIT parameter regime.

The basic plasma model described by Braams ${ }^{1}$ involves solving the continuity equation, the parallel momentum balance equation, and separate energy balance equations for the ions and the electrons. If multiple ion species are present, they are all assumed to have a common temperature, but their densities and parallel velocities are solved for using additional continuity and parallel momentum balance equations for each species. Momentum and heat transport parallel to the magnetic field, B, are given by the classical collisional theory ${ }^{2}$. On the other hand, transport perpendicular to $\mathbf{B}$ is represented by anomalous diffusion coefficients which are adjusted to agree with experimental measurements. These transport coefficients are generally taken to be constant in radius and poloidal angle, although this is not necessary.

The goal of our work has been to include both the classical cross-field drift terms and the effects of parallel currents in the equations used in the B2 code. The motivation for including the cross-field terms comes from simple model calculations ${ }^{3}$ which indicate that the classical flows can contribute an important asymmetry which may help explain the transition from L-mode to H-mode confinement. Radial electric fields which arise near the separatrix cause $\mathbf{E} \times \mathbf{B}$ poloidal rotation which may also be related to the L-to- $\mathrm{H}$ mode transition through its effect on edge tubulence. .,5 $^{4}$ Including the parallel currents is done to provide a tool for understanding the biased divertor experiments ${ }^{6}$ on DIII-D at General Atomics. Such biasing may provide an effective means of controlling the asymmetry of the power flow to different divertor plates. Furthermore, even for tokamaks with grounded plates, asymmetries drive parallel currents.

\section{THE MODEL}

\section{Toroidal Geometry}

Because the cross-field drifts depend on the direction of the magnetic field relative to spatial gradients, care needs to be taken in defining the geometry and coordinate system. The convention used is that in viewing the plasma poloidal cross-section, the 
magnetic axis is to the left as shown in Fig. 1. The toroidal unit vector, $\hat{\mathrm{e}}_{\phi}$, is taken into the paper, and the toroidal plasma current is always in this same direction. Thus, the poloidal magnetic tield, $B_{p} \hat{\mathbf{e}}_{p}=\hat{\mathbf{e}}_{\phi} \times \nabla \psi / R$, is directed in the clockwise direction inside the separatrix. Here, $\psi$ is the poloidal flux function, $\mathrm{R}$ is the major radius, and we always take $B_{p}$ to be positive. On the other hand, for the toroidal magnetic field, $B_{\phi} \hat{\mathbf{e}}_{\phi}, B_{\phi}$ may be positive or negative. For $B_{\phi}$ positive, the toroidal field is into the paper, and the $\mathbf{B} \times \nabla B$ ion drift is directed toward the upper $\mathrm{x}$-point in Fig. 1.

We use a right-handed coordinate system with unit vectors $\left(\hat{\mathbf{e}}_{r}, \hat{\mathbf{e}}_{f}, \hat{\mathbf{e}}_{\|}\right)$. Here $\hat{\mathbf{e}}_{\|}$ is the unit vector along the magnetic field, $\hat{\mathbf{e}}_{r}=\nabla \psi /|\nabla \psi|$ is the radial-like direction, and $\hat{\mathbf{e}}_{f}=\hat{\mathbf{e}}_{\|} \times \hat{\mathbf{e}}_{r}$ lies in the flux surface. Note that $\hat{\mathbf{e}}_{r}$ always points to the right at the outer scrap-off layer, but that $\hat{\mathbf{e}}_{f}$ and $\hat{\mathbf{e}}_{\|}$change direction with the sign of $B_{\phi}$. The relationship between the various unit vectors car be summarized as follows:

$$
\hat{\mathbf{e}}_{f}=\frac{B_{\phi}}{B} \hat{\mathbf{e}}_{p}-\frac{B_{p}}{B} \hat{\mathbf{e}}_{\phi}
$$

and

$$
\hat{\mathbf{e}}_{\|}=\frac{B_{p}}{B} \hat{\mathbf{e}}_{p}+\frac{B_{\phi}}{B} \hat{\mathbf{e}}_{\phi}
$$

Here $B$ is the magnitude of $\mathbf{B}$ and is always positive. T While, transport in both the $\hat{\mathbf{e}}_{f}$ and $\hat{\mathbf{e}}_{\|}$directions have components in the poloidal and toroidal directions, only the poloidal components will be used explicitly in the equations here because toroidal symmetry is assumed. However, toroidal drifts will be calculated as a diagnostic.

\section{Equations for Cross-Field Flows}

We use the fluid equations ${ }^{2}$ to obtain the cross-field plasma flows. First we consider the fluid velocities as obtained from the perpendicular ion momentum equation in which inertia and viscosity are neglected:

$$
0=-\frac{\nabla_{\perp} p_{i}}{n e}+\mathbf{E}_{\perp}+\mathbf{v}_{\perp} \times \mathbf{B}-\eta_{\perp} \mathbf{J}_{\perp}
$$

where $\mathbf{E}_{\perp}$ is the perpendicular electric field, $\mathbf{v}_{\perp}$ is the perpendicular ion velocity, $p_{i}=$ $n T_{i}$ is the ion pressure, $n$ is the density, $T_{i}$ is the ion temperature, $e$ is the magnitude of the electron charge, $\mathbf{J}_{\perp}$ is the perpendicular plasma current, and $\eta_{\perp}$ is the perpendicular resistivity. An anomalous resistivity can be adcied as we shall mention later. The perpendicular drifts are obtained from Eq. (3) as

$$
\mathbf{v}_{\perp}=\frac{\left(\mathbf{E}_{\perp}-\nabla_{\perp} p_{i} / n e\right) \times \mathbf{B}}{B^{2}}-\eta_{\perp} \frac{\mathbf{J}_{\perp} \times \mathbf{B}}{B^{2}}
$$

The perpendicular currents are found by adding the electron and ion momemtum equations with inertia and viscosity terms neglected. This procedure yields the standard relation

$$
\mathbf{J}_{\perp}=\frac{\mathbf{B} \times \nabla_{\perp} p}{B^{2}}
$$


where $p=p_{e}+p_{i}$ is the total plasma pressure. Combining Eqs. (3-4) then gives

$$
\mathbf{v}=\frac{\left(\mathrm{E}-\nabla p_{i} / n e\right) \times \mathrm{B}}{B^{2}}-\eta_{\perp} \frac{\nabla \perp p}{B^{2}}
$$

The diffusive term that has been used in the $\mathrm{B} 2$ code is represer tative of an anomalous contribution to the last terms in Eq. (6), although it is generally taken to be proportional to $\nabla n$ rather than $\nabla p$.

The $\mathrm{B} 2$ code solves the fluid equations in the poloidal plane using the coordinates $(x, y)$ to correspond to the $\hat{\mathbf{e}}_{p}$ and $\hat{\mathbf{e}}_{r}$ directions, respectively. Using Eqs. $(1,2,6)$, one can write the drift terms explicitly in the $(x, y)$ coordinates as

$$
v_{x}=\frac{B_{\phi}}{B^{2}}\left(\frac{\partial \phi}{\partial y}+\frac{1}{n \epsilon} \cdot \frac{\partial p_{i}}{\partial y}-\eta_{\perp} \frac{B_{\phi}}{B^{2}} \frac{\partial p}{\partial x}\right)+\frac{B_{p}}{B} v_{\|}
$$

and

$$
v_{y}=-\frac{B_{\phi}}{B^{2}}\left(\frac{\partial \phi}{\partial x}+\frac{1}{n e} \frac{\partial p_{i}}{\partial x}+\eta_{\perp} \frac{1}{B_{\phi}} \frac{\partial p}{\partial y}\right)-\frac{D_{a}}{n} \frac{\partial n}{\partial y}+v_{a}
$$

Here $\mathrm{E}=-\nabla \phi$, and $v_{\|}$is the parallel velocity already calculated in the code. The last two terms in Eq. (8) have been used previously in the code to represent anomalous fluxes; $D_{a}$ is the anomalous diffusion coefficient, and $v_{a}$ is the anomalous convective velocity. The values of the $D_{a}$ and $v_{a}$ used for tokamak edge plasma modeling are typically sufficient to make the classical terms proportional to $\eta_{\text {.L }}$ unimportant.

It should be mentioned that B2 represents the solution on a grid $\left(x_{0}, y_{0}\right)$ in which $x_{0}$ is the distance along a particular flux surface, and $y_{0}$ is radial-like distance normal to the flux surface at a given value of $x$ (we use the midplane, $x=0$ ). Metric factors $\left(h_{x}, h_{y}\right)$ are required to translate to the physical location $(x, y)$. Thus,

$$
\frac{\partial}{\partial x} \rightarrow \frac{1}{h_{x}(x, y)} \frac{\partial}{\partial x_{0}}
$$

and likewise for the $y$-coordinate.

In addition to ion fluid drifts, we include the cross-field energy flux ${ }^{2}$ for both the ions and the electrons. For the ions, this term has the form

$$
q_{c f}=-\frac{5}{2} \frac{n T_{i}}{e B^{2}} \mathbf{B} \times \nabla T_{i}
$$

while for electrons, the overall sign changes to a plus. In terms of the $(x, y)$ coordinates, we have

$$
q_{c f x}=-\frac{5}{2} \frac{n T_{i} B_{\phi}}{e B^{2}} \frac{\partial T_{i}}{\partial y}
$$

and

$$
q_{c f y}=\frac{5}{2} \frac{n T_{i} B_{\phi}}{e B^{2}} \frac{\partial T_{i}}{\partial x}
$$

and likewise for the electrons with a sign change. 
There are also corrections to the electron energy balance equation owing to the presence of the parallel current, $J_{\|}=n c\left(v_{\|_{i}}-v_{\|_{e}}\right)$, along $\mathbf{B}$. One term is the effect of Joule heating, and is represented by adding a $\mathbf{J} \cdot \mathbf{E}$ source term. The second arises to account for the difference in the electron and ion velocities in the convected heat flux. We use the convention that the parallel velocity used to convect energy in both ion and electron energy balance equations is the ion parallel velocity. Thus, the electron heat flux in the $x$-direction has a new term given by

$$
q_{x e j}=-\left(\frac{5}{2}+\alpha\right) \frac{B_{p}}{B} \frac{T_{e} J_{\|}}{e}
$$

where the term proportional to $\alpha\left(=0.71\right.$ for $\left.Z_{\text {eff }}=1\right)$ comes from the thermal force correction. The current modification given by Eq. (12) generally dominants over Joule heating for the cases we have studied. There are also corrections to the perpendicular heat flux from these same effects, but we have neglected them as small for now.

\section{Equations for Electrostatic Potential and Parallel Current}

Some of the correction terms we have discussed depend on the electrostatic potential and the parallel current. These quantities are obtained from two equations: the inertialess parallel electron rnomentum equation written in the form

$$
0=-\frac{1}{n e} \frac{\partial p_{e}}{\partial x}+\frac{\partial \phi}{\partial x}-\frac{\alpha}{e} \frac{\partial T_{e}}{\partial x}+\frac{B}{B_{p}} \eta_{\|} J_{\|}
$$

where $\eta_{\|}$is the parallel resistivity, and the current continuity equation, $\nabla \cdot \mathbf{J}=0$, written as

$$
B_{p} \frac{\partial}{\partial x}\left(\frac{1}{B} J_{\|}\right)=-\nabla_{\perp} \cdot \mathbf{J}_{\perp}
$$

Substituting for $J_{\|}$from Eq. (13) and for $\mathbf{J}_{\perp}$ from Eq. (5) then gives an equation which solve for $\phi$ in the scrape-off region (SOL) given boundary conditions on the divertor plates. These boundary conditions come from the analysis discussed in Refs. 7 and 8 .

The solution of the potential equation in the core region of the plasma is complicated by the need to impose periodic boundary conditions. If we add an anomalous perpendicular current to Eq. (5) proportional to $\left(\mathbf{E}_{\perp}+\nabla_{\perp} p_{e} / n e\right)$ as suggested by $B$. J. Braams (private communication), the equation for $\phi$ becomes two-dimensional. We are then able to find a potential in the core region as well. However, the connection of this ansatz to a physical mechanism needs to be determined, so we shall present results where the potential is celculated from Eq.(14) in the SOL, while in the core, the potential is set to the value just across the separatrix in the SOL.

\section{SIMULATION RESULTS}

We have modeled two types of configurations to study the effects of with the crossfield terms and parallel current. One is a double-null divertor of the type planned for 
CIT as shown in Fig. 1. We have simulated the outer portion of the divertor to study up-down asymmetries arising from the cross-field drifts. Imaginary lines (shown as dots in Fig. 1) are drawn normal to the flux surfaces through the x-points. These lines are taken as reflecting boundaries following previous CIT calculations by Braams and Stotler, who, by symmetry, only consider the lower half of the outer scrape-off layer. The reflecting boundary conditions modify the physics in the vicinity of the $x$-point, so for this model, we are only partially describing the influence of the cross-field drifts. Nevertheless, the model does give insight into the asymmetries causes by the drifts.

The full problem is to include the inside scrape-off layer as well, and apply periodic boundary conditions in the core region. We have made the first step toward this proper treatment of the $\mathrm{x}$-point region by considering a single-null geometry as shown in Fig. 2, and applying periodic boundary conditions in the core. These calculations model typical DIII-D operation. The calculations show the influence of the periodic boundary conditions and the strong effect that biasing can have on divertor plate asyinmetries.

\section{Double-Null Configuration, Outer SOL Only}

To model the CIT magnetic field configuration, we use poloidal flux surfaces generated from a conformal mapping procedure by B. J. Braams (private communication, Sept. 1990) for the new $R=2.5 \mathrm{~m}, B=9 \mathrm{~T}$ CIT design. The flux surfaces in the outer lower quadrant are shown in Fig. 3 . We reflect this configuration vertically, about the midplane to obtain the outer upper quadrant as well. We thus include both the upper and lower divertor plates on the outside, and can study asymmetries. The toroidal field, $B_{\phi}$, is positive, so the $\mathbf{B} \times \nabla B$ ion drift is toward the upper x-point.

Because no particle or energy flux is allowed to flow across the boundaries through the $\mathrm{x}$-point, there is a problem with the cross-field fluxes which depend on gradients along the boundary rather than normal to it as for the other fluxes. Consequently, we have multiplied the cross-field fluxes by a function which goes to zero smoothly over the last few grid points before the reflecting boundary. The other choice is to let the normal fluxes become nonzero at the boundary to cancel the cross-field fluxes. While both are clearly unphysical, the former method allows convergent solutions while the latter is more problematic.

We have performed a set of simulations with the cross-field fluxes present to determine the asymmetries that result. The $\mathrm{B} 2$ code is run using boundary conditions on the innermost core $f$ ux surface of consiant density and temperature; the density is always set at $5 \times 10^{19} \mathrm{~m}^{-3}$ while the temperature is varied between $250 \mathrm{eV}$ and 500 $\mathrm{eV}$. For the divertor plates, the parallel velocity is set equal to the local sound speed, and the energy transmission factors for the ions and electrons are set to 2.5 and 4 , respectively. The particle recycling coefficient on the plates is taken as 0.97 . On all other boundaries, zero particle flux boundary conditions are used, and the ion and electron temperatures are equal to $2 \mathrm{eV}$. The anomalous density diffusion coefficient is $D_{a}=1$ $\mathrm{m}^{2} / \mathrm{s}$, and a srrall convective velocity of $v_{a}=-10 \mathrm{~m} / \mathrm{s}$ is used. If we set $v_{a}=0$, the code has difficulty with convergence. However, doubling $v_{a}$ to $-20 \mathrm{~m} / \mathrm{s}$ does not change the calculated profiles by more that $\approx 20 \%$ except very near the wall $\left(y=y_{\text {ma: }}\right)$. For 
the anomalous eneigy diffusion coefficients in the $y$-direction, $x_{y i}=\chi_{y c}=2 \mathrm{~m}^{2} / \mathrm{s}$ are used. The $(x, y)$ grid is taken to have $(96,24)$ cells. The distance between the $x$-point and the plate, $I_{x p}$, is $28 \mathrm{~cm}$.

In Fig. 4, we show the results of the total power on the upper and lower divertor plates on the outside for a range of core temperatures. The asymmetry shown here increases with temperature because the cross-field terms increase with temperature and because the gradients become steeper. The higher power on the upper plate can be understood by the fact that the poloidal cross-field energy flux for the electrons [Eq. (10) with a minus sign] is directed toward the upper plate. The corresponding ion flux is in the opposite direction, but it does not cancel the electron term because the electron temperature gradient in the $y$-direction is larger than that for the ions.

The profile of the power striking the upper and lower plates for $T_{c}=500 \mathrm{eV}$ is shown in Fig. 5a. Note that the electron flux (long-dashed line) is particularly enhanced on the upper plate. The width of the profile is about $3 \mathrm{~cm}$ on the upper plate and 4.5 $\mathrm{cm}$ on the bottom. Also, the profile for the ion flux on the lower plate lower plate has a irregularity on it that indicates the grid resolution is not yet sufficient. If we increase $T_{c}$ even further, the gradients become too steep, and the code does not converge. For comparison, the power flux for the $T_{c}=500 \mathrm{eV}$ symmetric case with no cross-field drifts is shown Fig. 5b. Here each plate receives $31.1 \mathrm{MW}$.

In order to estimate the expected power to the outer plates for CIT, we use the following: For $500 \mathrm{MW}$ of fusions power, there is $100 \mathrm{MW}$ of alpha-particle power, of which $75 \%$ is assumed to go to the outer SOL. Splitting this power between the upper and lower plates gives $37.5 \mathrm{MW}$ per plate, neglecting any loss from radiation. Thus, the $T_{c}=500 \mathrm{eV}$ case is in the required range.

Figure 6 shows the radial profiles at the midplane for the $T_{c}=500 \mathrm{eV}$ case. The temperatures and potential are given in Fig. 6a. Note that the potential is not calculated in the core region. The electron temperature falls more rapidly because of the large electron conductivity along $\mathbf{B}$; the ion temperature follows $T_{e}$ because of collisional coupling. The density profile in Fig. $6 \mathrm{~b}$ shows a characteristic slower decay. Changing $v_{a}$ to $-20 \mathrm{~m} / \mathrm{s}$ decreases the magnitude of the density scale length at the separatrix by $\approx 20 \%$, but the density at the wall $\left(y_{0}=0.072 \mathrm{~m}\right)$ decreases from $5 \times 10^{18} \mathrm{~m}^{3}$ to $2 \times 10^{18} \mathrm{~m}^{3}$.

A second effect of the cross-field drifts is that a significant toroidal velocity develops while the poloidal velocity remains small. The $\mathbf{E} \times \mathbf{B}$ and diamagnetic drifts from Eq. (5) both give an ion velocity directed toward the upper plate, in the - $\hat{\mathbf{e}}_{f}$ direction. However, a substantial parallel velocity develops which results in a pcloidal velocity which is roughly an order of magnitude smaller than the cross-field velocities. If we let $v_{f c}$ stand for the cross-fieid velocity in the $\hat{\mathbf{e}}_{f}$ direction, then from Eqs. (1-2), the poloidal velocity, $v_{p}$, is

$$
v_{p}=v_{f c} \frac{B_{\phi}}{B}+v_{\|} \frac{B_{p}}{B} .
$$

What happens is that a positive $v_{\|}$develops to nearly balance the term from $v_{f c}$. The poloidal velocity needs to stay small because it is a measure of the particle flux into the plate, which changes little. Since the parallel direction is mostly along the toroidal 
direction, a toroidal drift results, given by

$$
v_{t o r}=-v_{f c} \frac{B_{p}}{B}+v_{\|} \frac{B_{\phi}}{B}
$$

Figure 7 shows contour plots of the various velocities in the regions between the $x$ points; the abscissa is the poloidal-like coordinate $x_{0}$ and the ordinate is the radial-like coordinate $y_{0}$. The separatrix is located at $y_{0}=0$. For reference, the ion-acoustic velocity at $100 \mathrm{eV}$ for deuterium is about $10^{5} \mathrm{~m} / \mathrm{s}$, and the toroidal velocity near the separatrix is about $10 \%-20 \%$ of that.

These results indicate that the cross-field drifts do not result in a large power asymmetry on the plates, but before firm conclusions can be stated, parameter variations need to be made and the treatment of the $x$-point region improved to allow flow through this region. The asymmetries that do exist will drive parallel plasma currents; we are just beginning to look at this effect. The potential needs to be calculated in the core region which also may influence the conclusions.

\section{Single-Null Configuration with Bias Voltage}

For the single-null configiration shown in Fig. 2, we have removed the artificial boundary in B2 that passed through the $\mathrm{x}$-point, which prevented flow near the $\mathrm{x}$-point in the poloidal direction; the core now has periodic boundary conditions and the private flux region is continuous. We shall show the strong effect that divertor plate biasing ${ }^{6}$ can have on controlling the distribution of power between the inner and outer plates.

For this study, we use a DIII-D configuration with the following input parameters:

$$
\begin{aligned}
& D_{a}=2 \mathrm{~m}^{2} / \mathrm{s} \\
& \chi_{y e}=4 \mathrm{~m}^{2} / \mathrm{s} \\
& T_{e, \text { core }}=100 \mathrm{eV} \\
& n_{\text {core }}=2 \times 10^{19} \mathrm{~m}^{-3}
\end{aligned}
$$

$$
\begin{aligned}
& v_{a}=-40 \mathrm{~m} / \mathrm{s} \\
& \chi_{y i}=0.2 \mathrm{~m}^{2} / \mathrm{s} \\
& T_{i, \text { core }}=100 \mathrm{eV} \\
& \Gamma_{\text {recycle }}=0.97
\end{aligned}
$$

These parameters result in a total power of $\sim 1 \mathrm{MW}$ striking the divertor plates.. The $(x, y)$ grid has $(32,16)$ cells, which is somewhat coarse but useful for initial computational efficiency.

The poloidal current is plotted versus the bias voltage in Fig. 8. Note the rather sensitive behavior at small voltages. This curve is similar to the $I \propto \tanh (-e \phi / T)$ characteristic for a double probe. However, here the situation is more complicated as the temperatures at the two ends change strongly with $\phi_{b}$. The current in Fig. 8 at about $50 \%$ of the saturation current for both $\phi_{b}= \pm 50 \mathrm{~V}$. We tried running the code for $\phi_{b}=100 \mathrm{~V}$, but it did not converge.

A more striking result is shown in Fig. 9 where the power to each plate is given, together with that prcvided from the bias supply. The power to each plate can be varied by a factor of 4 , and the power ratio by a factor of 16 . Note that a very modest bias supply power is needed except at the largest voltages.

The power asymmetry can be explained by the large electron convective heat flux that arises in response to the bias potential. This additional heat flux can be written 
in the form given by Eq. (12) if one adopts the convention of using the ion velocity for the remaining electron convective heat flow, $(5 / 2) n_{e} T_{e} v_{i}$. In terms of the poloidal current density, $J_{x}$, the additional heat fiux is,

$$
q_{x e j}=3.2 J_{x} T_{e} / e
$$

where $\alpha=0.7$ has been used. The total power associated with this flow can be estimated as $-3.2 I_{x} T_{e} / e$, where $I_{x}$ is the polodial current in Fig. 8. Thus, for $\phi_{b}=-5$ $\mathrm{V}, I_{x} \approx 2 \mathrm{kA}$, and for an average value of $T_{e}=50 \mathrm{eV}$, the extra electron convected power is $-0.3 \mathrm{MW}$. The minus sign means this power is directed toward the inner plate. By considering Fig. 9, one can see that this power is of sufficient size, and of the proper sign, to account for the shift of power to the inner divertor plate for $\phi_{b}=-5 \mathrm{~V}$.

Finally, we note that the cross-field drifts were left off for the DIII-D cases discussed above. If we turn on the diamagnetic particle and energy fluxes given in Eqs. (7-8,1011 ), we calculate only $\sim 10 \%$ difference in the results. Presently, the code has trouble operating with the $\mathbf{E} \times \mathbf{B}$ velocities on, so we cannot make specific statements about that. Part of the problem concerns the resulting drifts near the $x$-point and how to extend them smoothly into the core region. We are presently working on this difficulty.

\section{SUMMARY}

We summarize our work to date by the following points:

1. Initial results indicate that cross-field drifts give asymmetries of $\sim 30 \%$ for CIT parameters assuming $500 \mathrm{MW}$ of fusion power. The asymmetries increase with power to the divertor plates.

2. The results may be affected by the treatment of the $\mathrm{x}$-point region for double-null configurations which needs to be upgraded by removing the artificial reflecting boundary as we have done for the single null. We will work on that this year.

3. The cross-field drifts do result in a toroidal rotation velocity in the SOL of $\sim 0.1 C_{s}$ while the poloidal velocity is constrained to a lower value.

4. We have demonstrated that a small bias-supply power can control the distribution of a large divertor power between inner and outer plates for the single null DIII-D parameter regime.

5. Dominant effect for power control is the additional electron parallel convective heat flux proportional to $J_{\|}$.

6. Power distribution control should also be possible for higher power, double null configurations like ITER or CIT, but simulations are yet to be done. This mechanism would provide a powerful technique for correcting asymmetries that arise from cross-field drifts and unbalanced MHD equilibria. 
7. Calculation of the potential in the core region to give proper $\mathbf{E} \times \mathbf{B}$ drifts remains to be done.

\section{ACKNOWLEDGEMENTS}

It is a pleasure to thank Drs. B. J. Braams, D. N. Hill, W. M. Nevins, and D. P. Stotler for many useful discussions. In addition to the discussions, Dr. Braams has provided us with a version of the $\mathrm{B} 2$ code and a magnetic configuration for CIT.

\section{REFERENCES}

1. B. J. Braams, "A Multi-Fluid Code for Simulation of the Edge Plasma in Tokamaks", NET Report, Nr. 68, January, 1987.

2. For example, S. I. Braginskii, in Reviews of Plasma Physics, edited by M. A. Leontovich (Consultants Bureau, New York, 1965), Vol. I, p. 205.

3. F. L. Hinton and G. M. Staebler, "Collisional Transport near a Separatrix and the H-Mode," Nucl. Fusion 29, 405 (1989).

4. K. C. Shaing and E. C. Crume, Jr., "Bifurcation Theory of Polodial Rotation in Tokamaks: A Model for the L-H Transition," Phys. Rev. Lett 63, 2369 (1989).

5. Y. B. Kim, P. H. Diamond, H. Biglari, and P. W. Terry, "Theory of ResistivityGradient-Driven Turbulence in a Differentially Rotating Plasma," Phys. Fluids B2, 2143 (1990).

6. R. D. Stambaugh, M. Ali Mahdavi, and M. Shimada, "Electrical Biasing of the Divertor Plate in Tokamaks," Bull. Am. Phys. Soc. 31, 1535 (1986).

7. G. M. Staebler and F. L. Hinton, "Currents in the Scrape-Off Layer of Diverted Tokamaks," Nucl. Fusion 29, 1820 (1989).

8. P. C. Stangeby, "Comment on Paper by Staebler and Hinton," Nucl. Fusion 30, 1153 (1990). 


\section{FIGURE CAPTIONS}

Fig. 1. Poloidal cross section of a tokamak like CIT with double-null divertor (four plates). The directions of various unit vectors are illustrated.

Fig. 2. Poloidal cross section of the DIII-D tokamak with a single-null divertor (two plates).

Fig. 3. Poloidal flux surfaces from a conformal mapping by B. J. Braams used to model the $R=2.6 \mathrm{~m}, B=9 \mathrm{~T}$ CIT configuration. We rotate these surfaces about the midplane to obtain the symmetric upper half region as well.

Fig. 4. Total power incident on the outer upper and lower divertor plates for CIT versus the assumed temperature on the core boundary just inside the separatrix. The asymmetry is caused by the presence of cross-field fluxes.

Fig. 5. Energy fluxes (total, ion, and electron) versus distance along the divertor plate for (a), the upper and lower divertor plates with $T_{c}=500 \mathrm{eV}$ with cross-field fluxes, and (b), the same case without cross-field terms.

Fig. 6. Midplane profiles of (a), potential, and ion and electron temperatures, and in (b), the density.

Fig. 7. Contour plots of various velocities in the core-SOL region showing a large toroidal velocity compared to the poloidal velocity for the $T_{c}=500 \mathrm{eV}$ case.

Fig. 8. The poloidal current versus bias voltage of the single null DIII-D case with the core temperature $T_{c}=100 \mathrm{eV}$.

Fig. 9. The power to the inner and outer divertor plates versus bias voltage, and the external power required from the bias supply. 


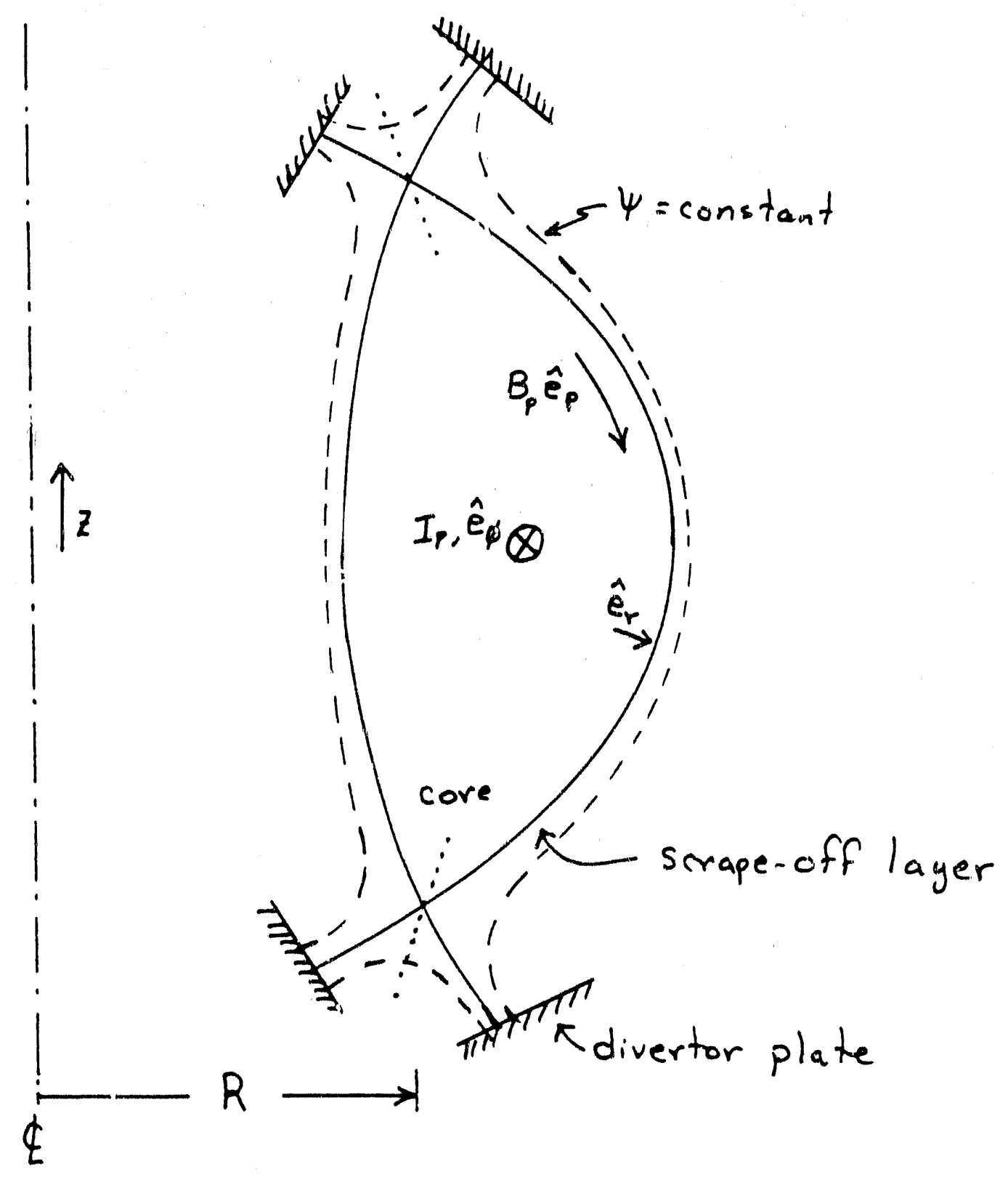

Fig. 1

11 


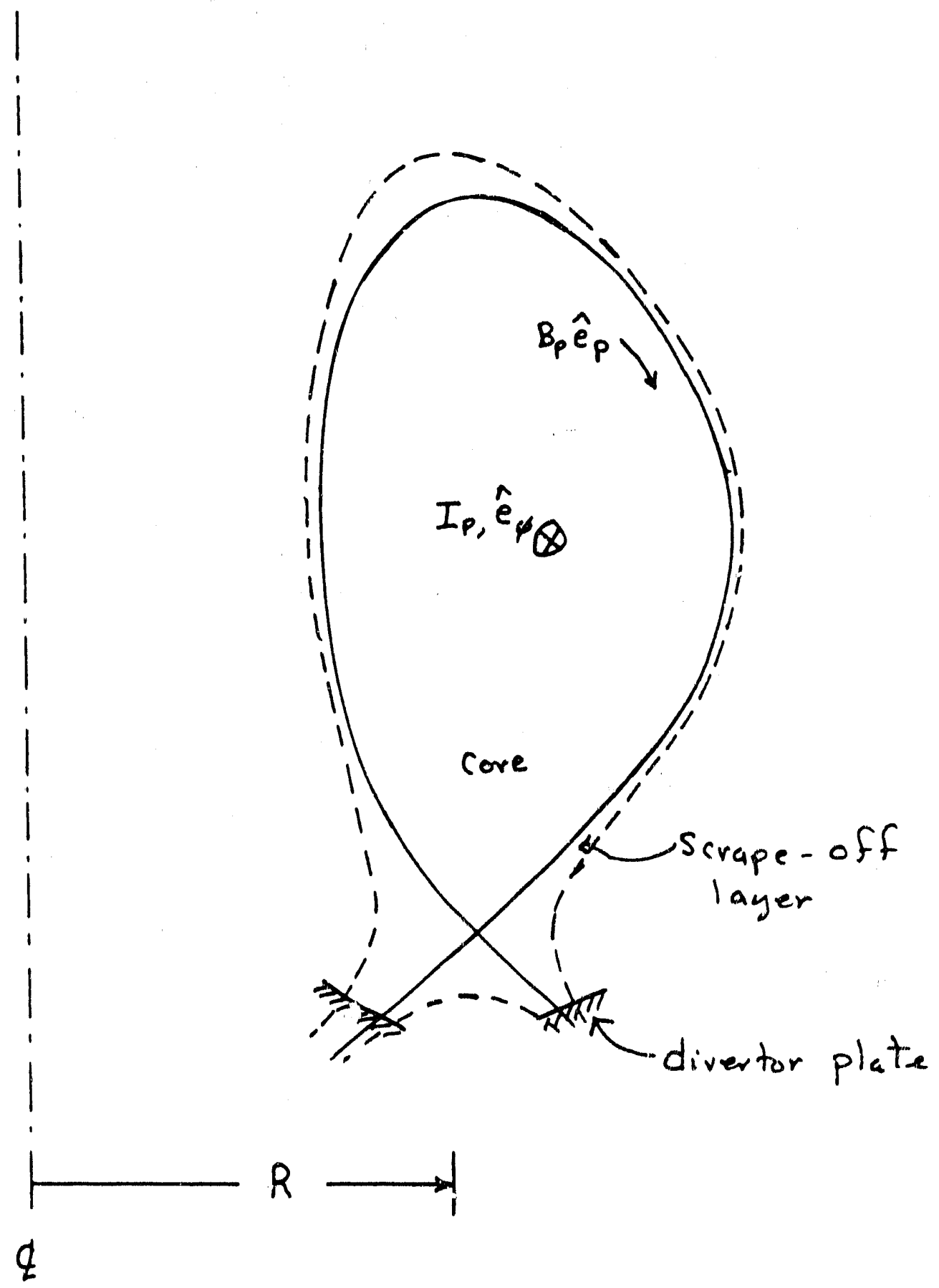

Fig. 2 


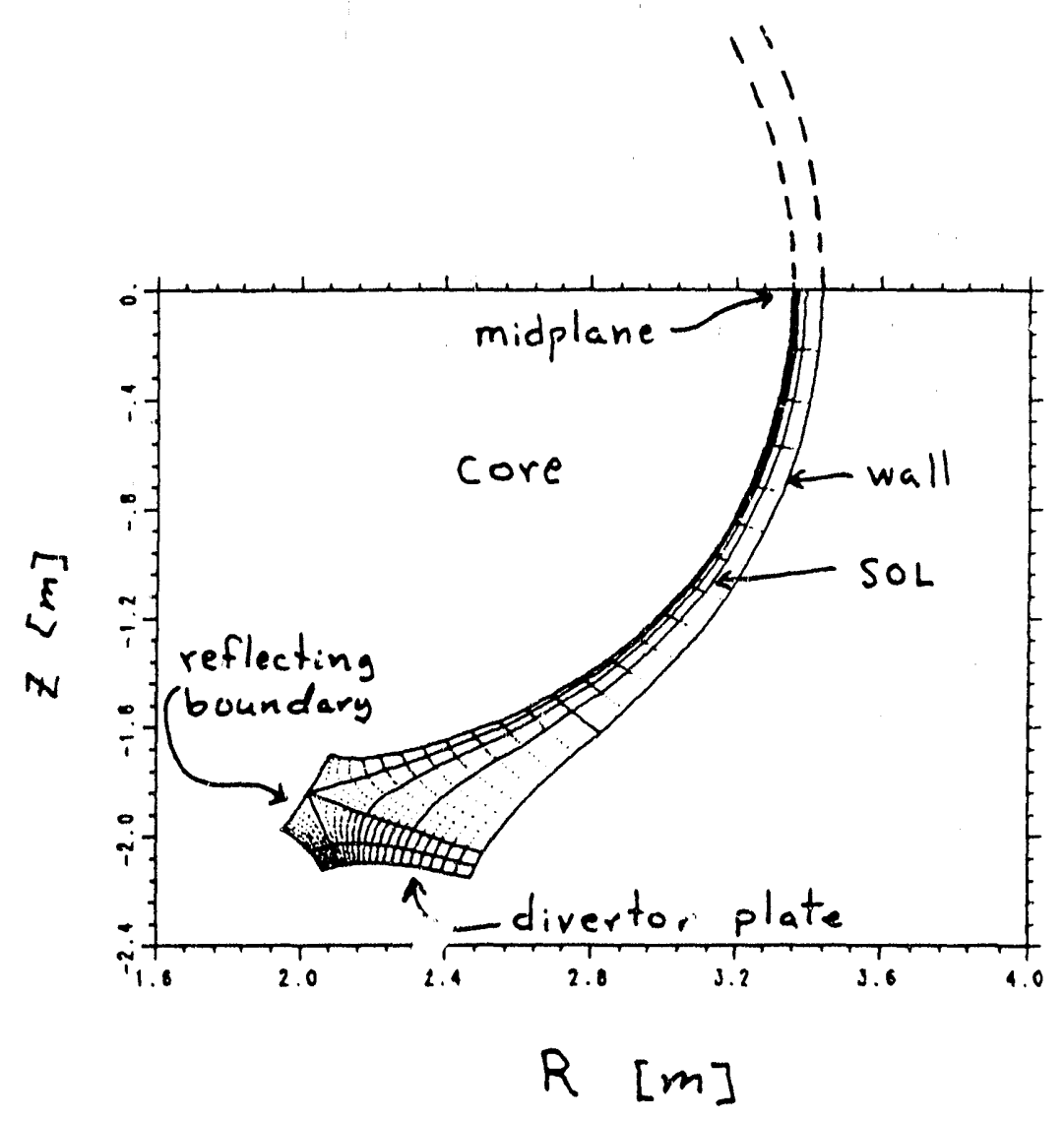

Fig. 3

13 


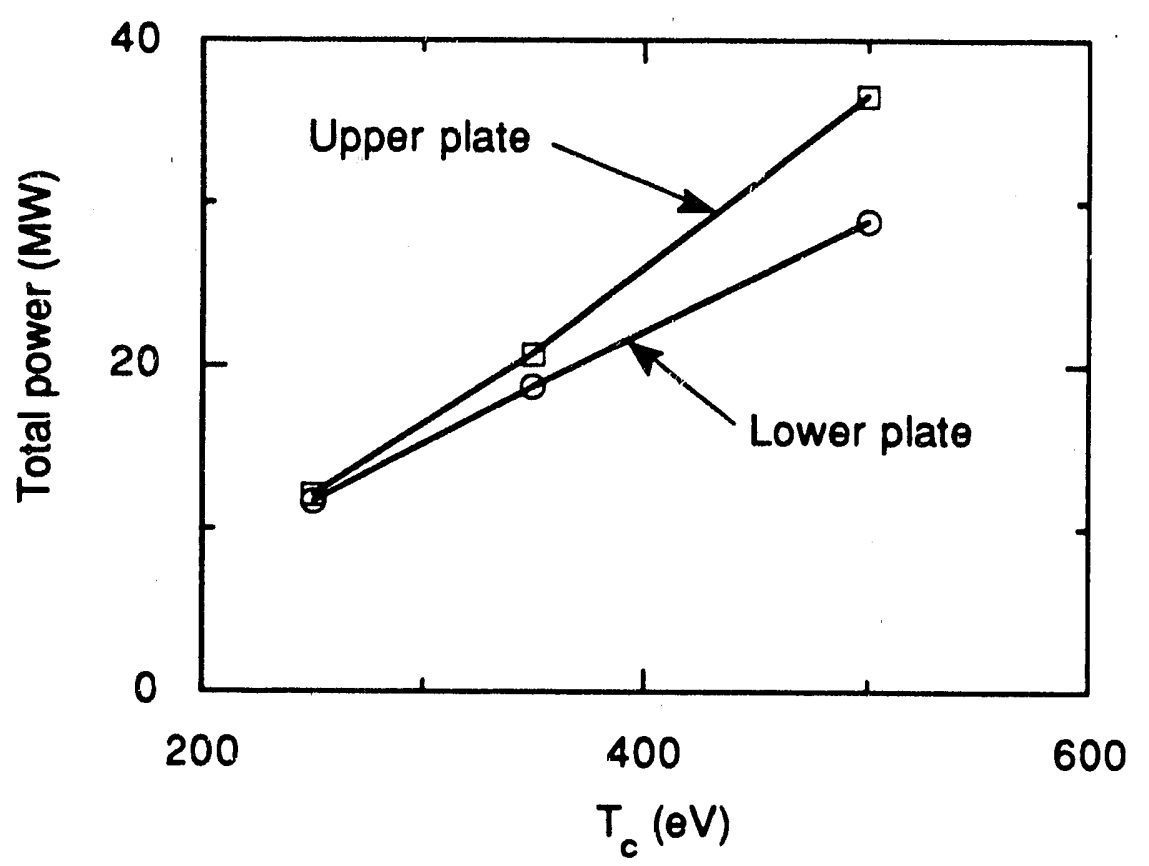

Fig. 4 
(a)
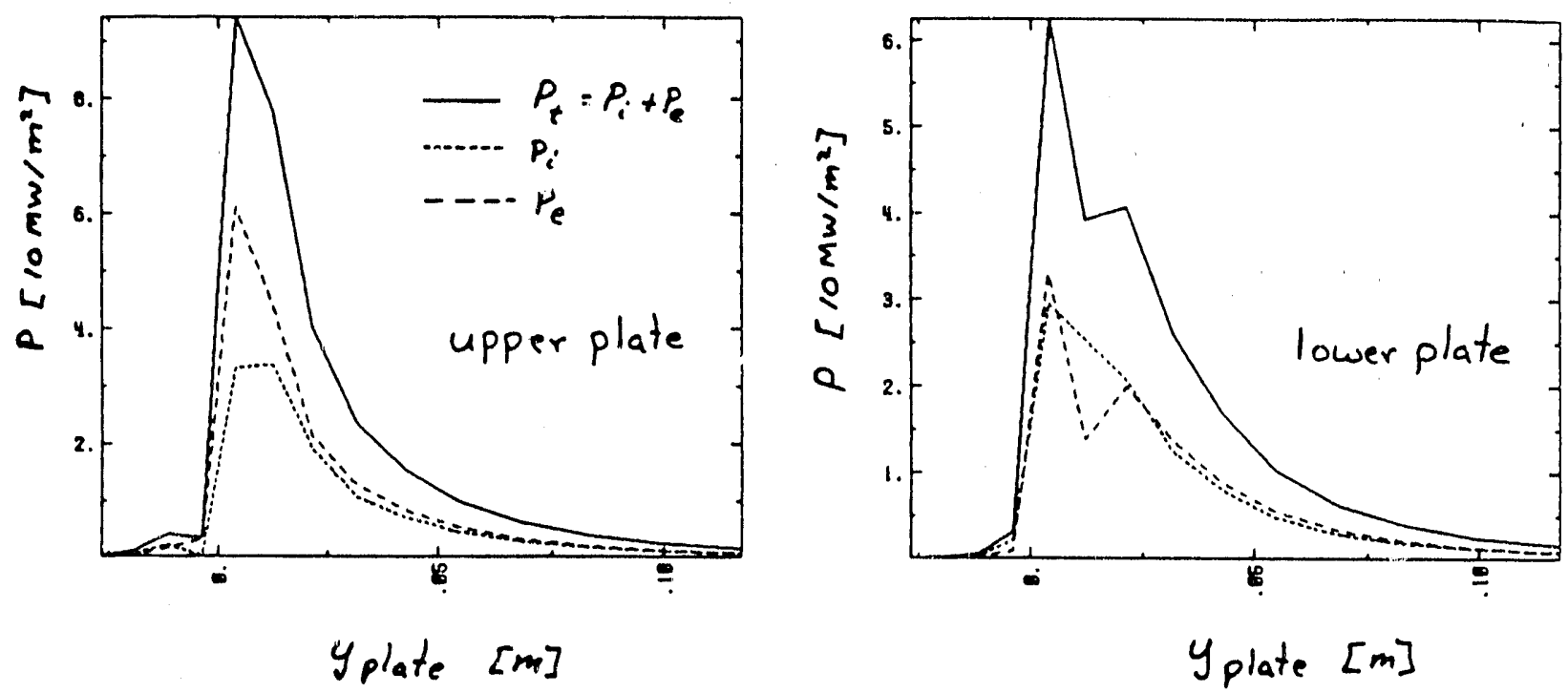

(b)

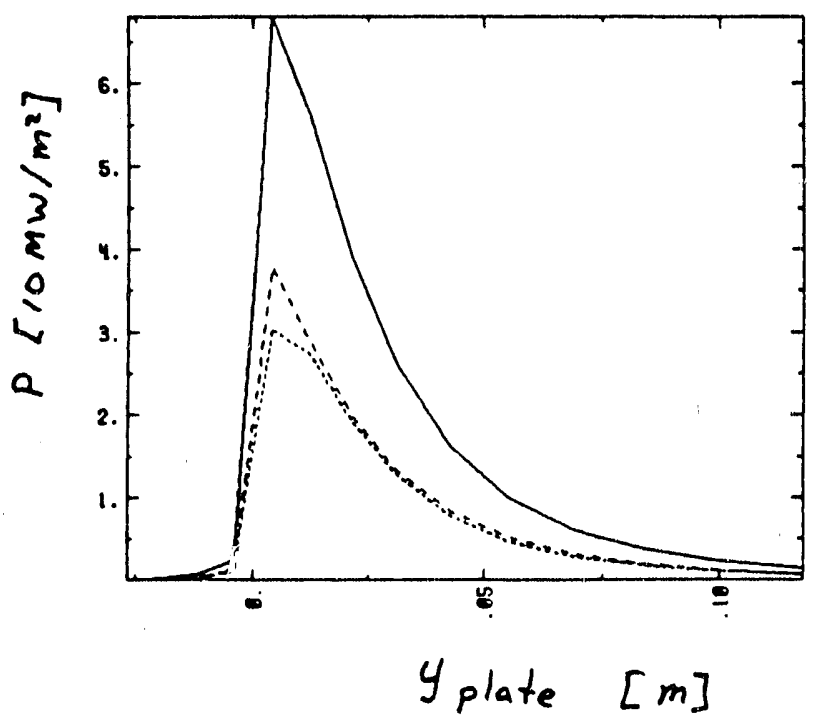

Fig. 5 


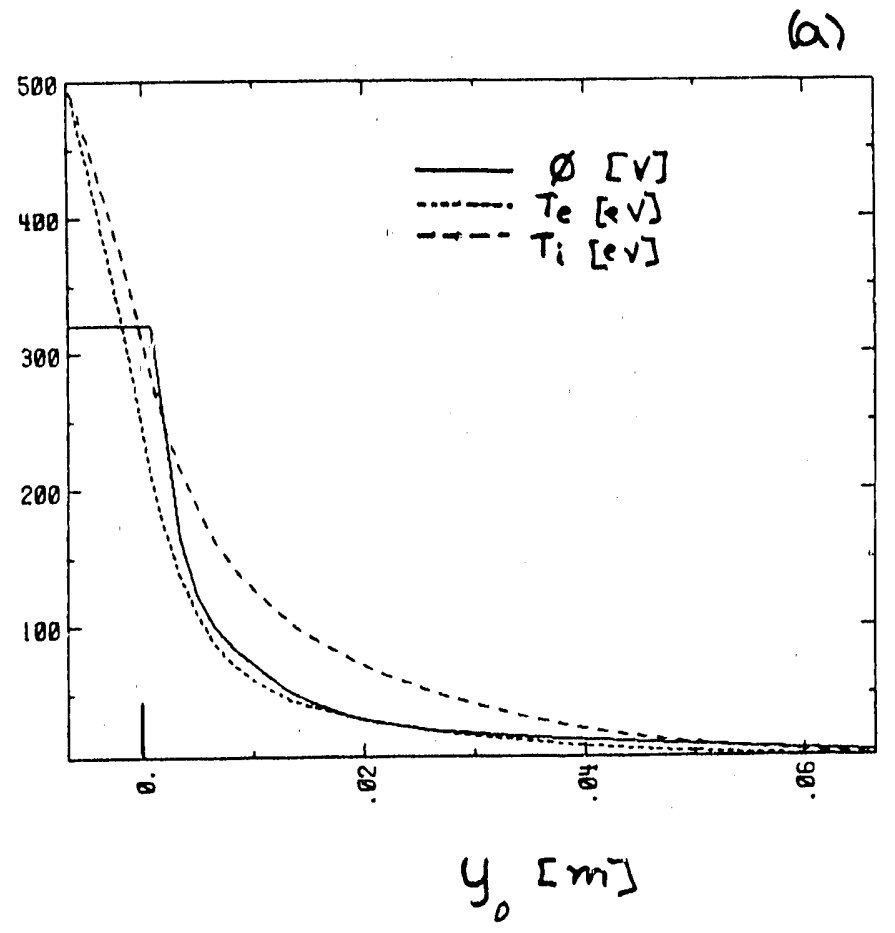

(b)

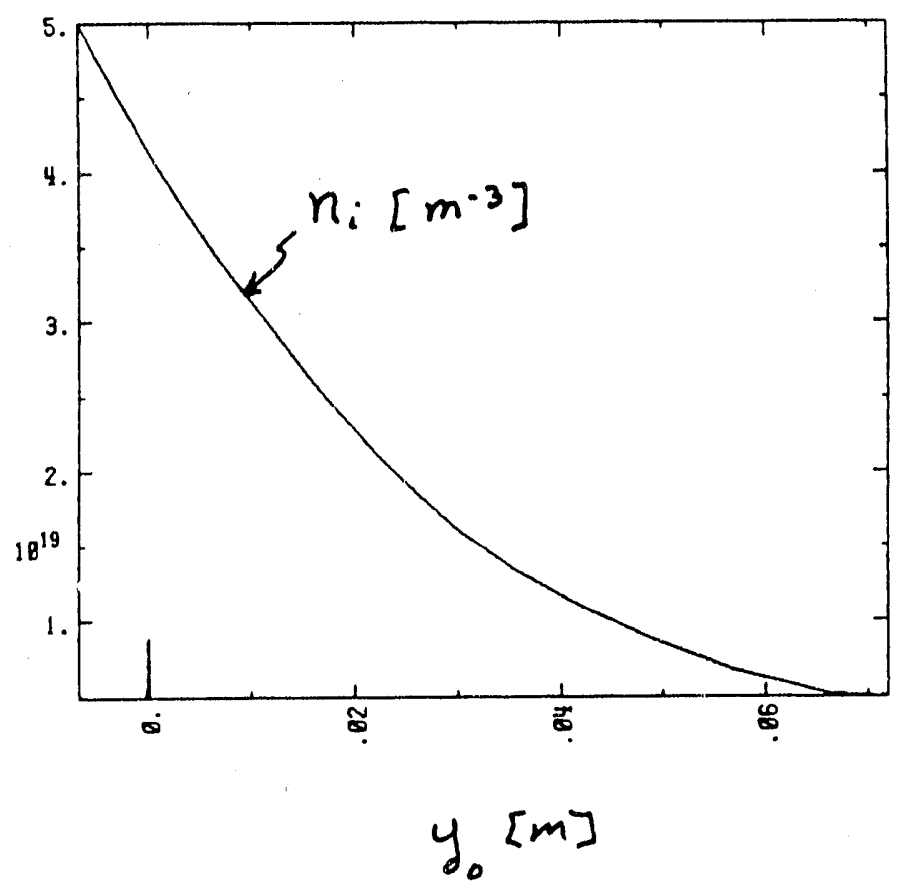

Fig. 6 

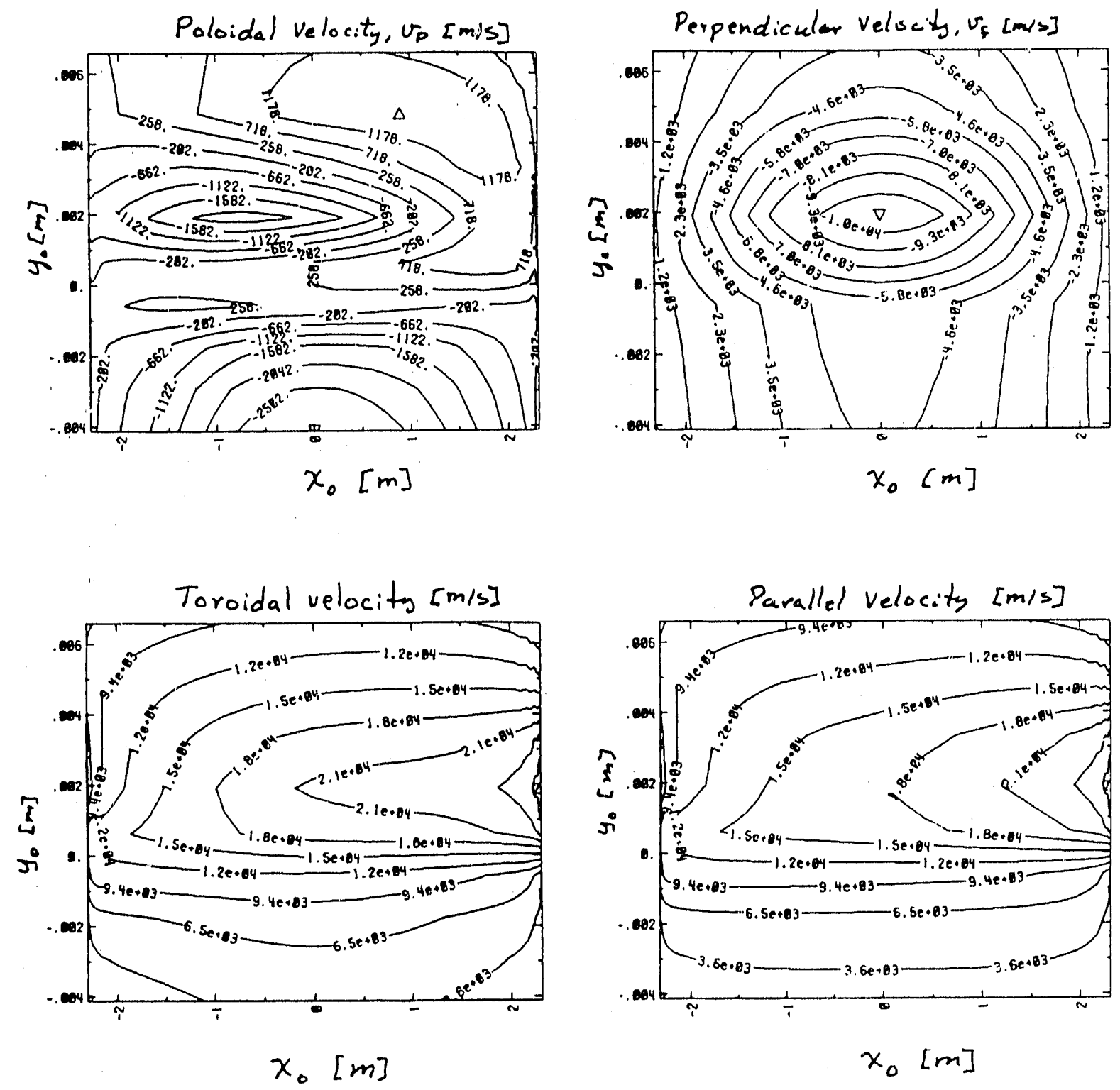

Fig. 7 


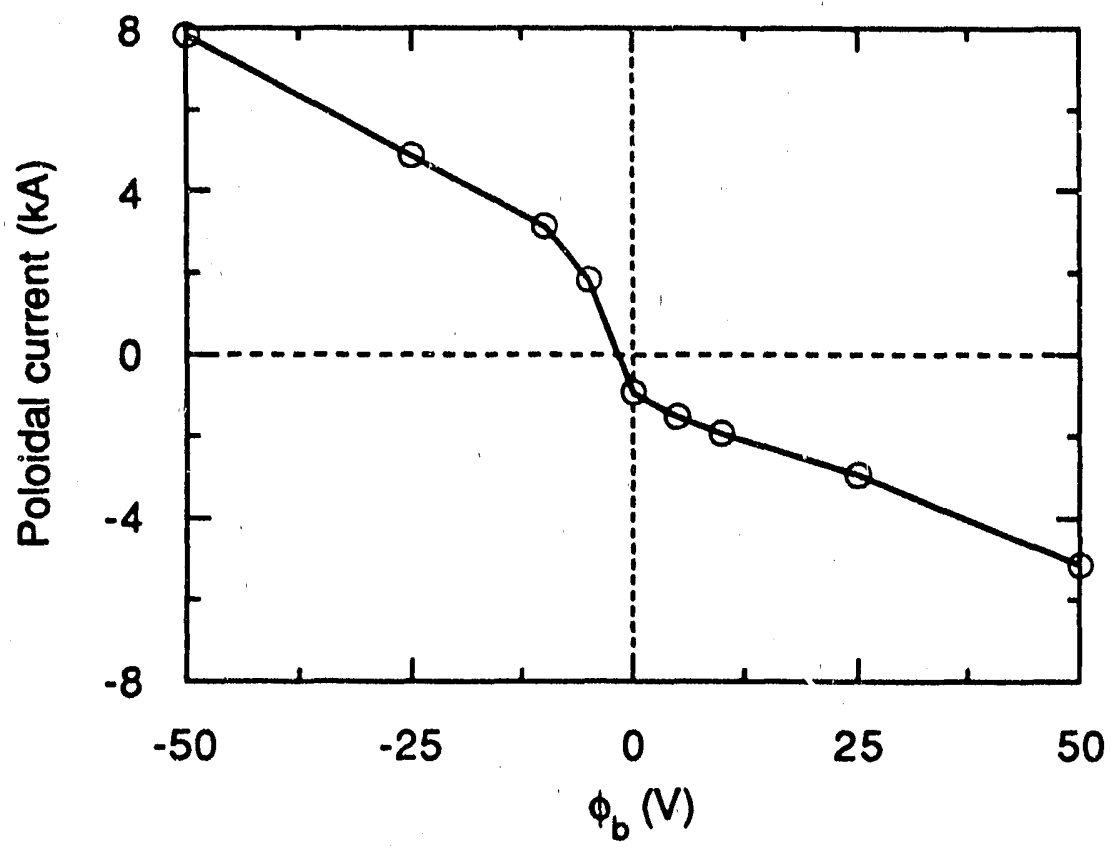

Fig. 8

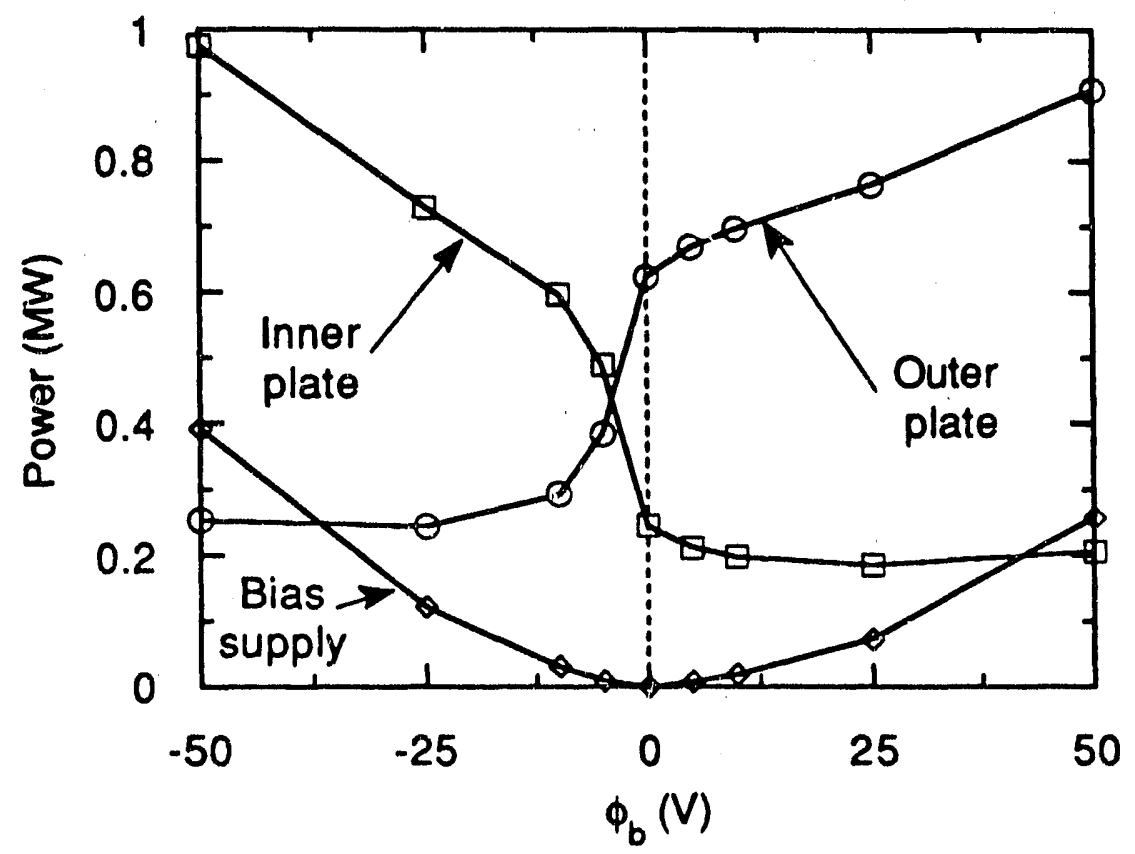

Fig. 9 

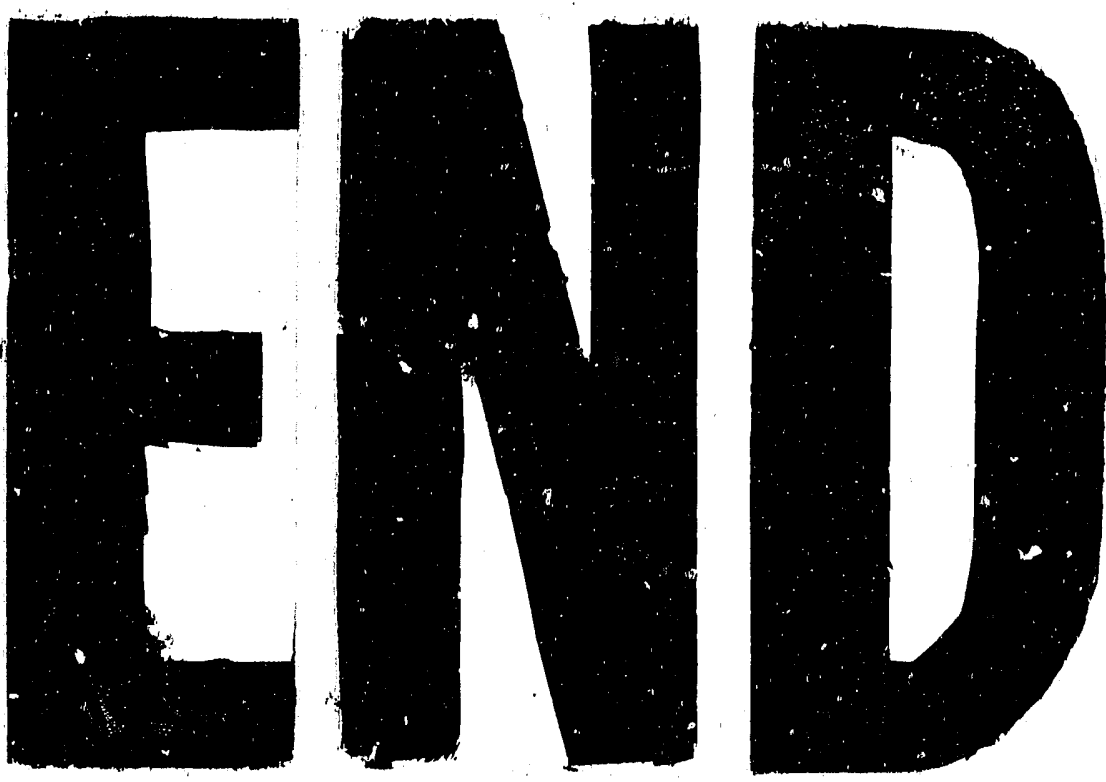

a
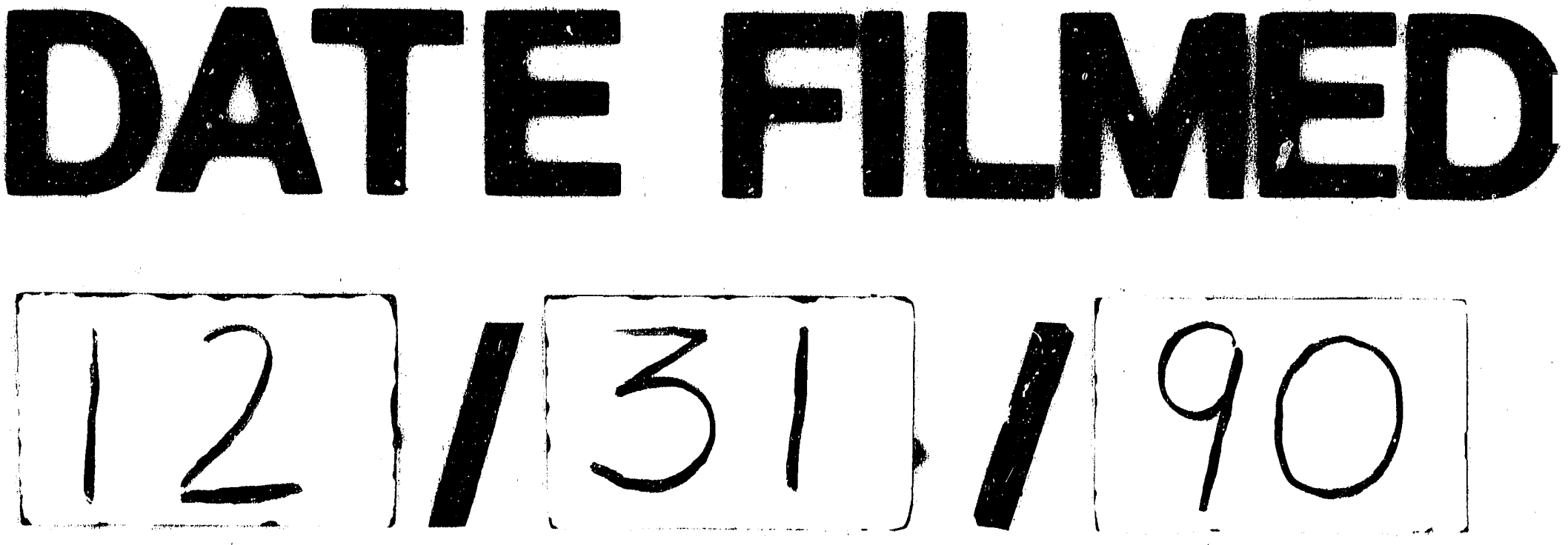
